Palmaris longus (PML) muscles of rhesus monkeys were transplanted, with or without anastomosis of the median nerve, to the nerve stump of the autograft. Because PML autografts revascularize spontaneously, vascular anastomoses were not performed. Muscle fibers regenerated in all autografts with neuroanastomosis, but in only three of eight autografts without neuroanastomosis. Five autografts without neuroanastomosis were replaced by noncontractile connective tissue. Growth and differentiation of muscle fibers into three fiber types and development of capillarity were analyzed histochemically, and succinate oxidase activity of whole-muscle homogenates was determined. None of these measures reached values for control PML muscles within 100 days of transplantation. In comparison to control muscles, autografts had slower times to peak tension and less absolute tension, but similar tension per square centimeter of muscle fiber cross-sectional area. Monkey PML autografts with neuroanastomosis were similar in structure and function to cat extensor digitorum longus autografts that had not had neuroanastomosis.

\section{NEUROANASTOMOSIS OF ORTHOTOPICALLY TRANSPLANTED PALMARIS LONGUS MUSCLES}

\author{
L. C. MAXWELL PhD, J. A. FAULKNER, PhD, \\ J. M. MARKLEY, JR., MD, and \\ D. R. WINBORN, BS
}

Since successful transplantation of entire mammalian skeletal muscles was first reported by Studitsky and Bosova, ${ }^{26}$ entire muscles of rats, ${ }^{2,29}$ cats, ${ }^{5,11,12,18,20}$ dogs, ${ }^{13}$ pigs $^{6}$ and humans ${ }^{8-10,25}$ have been successfully transplanted. Skeletal muscle transplantation has been employed clinically in humans in attempts to correct facial muscle paralysis $^{8,9,27}$ and anal incontinence. ${ }^{10,27}$ Since prior denervation is considered by most investigators to be essential to successful transplantation, ${ }^{9,10,26,27}$ most clinical and experimental work has made use of muscles transplanted two to four weeks after de-

From the Department of Physiology and the Department of Surgery, Section of Plastic Surgery. University of Michigan Medical School, Ann Arbor, MI.

Acknowledgment: This investigation was supported by a grant from the Muscular Dystrophy Association of America.

Address reprint requests to Dr. Maxwell at the Department of Physiology. University of Michigan Medical School, Ann Arbor, MI 48109.

Received for publication June 12, 1978; accepted for publication October 5. 1978

0148-639x/0201/0044 $\$ 00.00 / 0$

ه 1979 Houghton Miftlin Professional Publishers nervation. However, we have transplanted extensor digitorum longus muscles of cats both with and without prior denervation, and have found no benefit from prior denervation. ${ }^{\mathbf{5 . 1 8 . 2 0}}$ Similar findings have been reported for transplantation of rat, ${ }^{2.29} \mathrm{dog},{ }^{13}$ and monkey ${ }^{15}$ skeletal muscles.

Skeletal muscles of dogs have been transplanted with microneurovascular anastomosis performed at the time of surgery. ${ }^{13} A$ similar technique was used in two clinical cases by Harii et al ${ }^{8}$ for transplantation of human gracilis muscles to correct facial paralysis, and in one case by Schenck ${ }^{25}$ for transplantation of rectus abdominis muscle to repair forearm trauma. It is not clear whether neuroanastomoses, vascular anastomoses, or both are important to the successful transplantation of large muscle masses.

The purpose of this investigation was to determine the histochemical, biochemical, and contractile characteristics of palmaris longus muscles of rhesus monkeys after these muscles had been transplanted orthotopically with or without neuroanastomosis, and to compare the results with data obtained from control palmaris longus muscles. 


\section{METHODS}

Adult rhesus monkeys (Macaca mulatta) were anesthetized with $10 \mathrm{mg} / \mathrm{kg}$ ketamine administered intramuscularly, followed by intravenous injection of sodium pentobarbital. All surgical procedures were carricd out under strict sterile conditions. Entire palmaris longus muscles were exposed, and all neural, vascular, and tendinous connections were severed. The muscles were weighed under sterile conditions and were returned to their original orientation. The severed proximal and distal tendinous connections were sutured to the tendon stumps. In 9 of 17 autografts, fascicles of the severed palmaris longus motor branch of the median nerve were sutured to fascicles of the autograft motor nerve stump. Because of the small size of the vessels supplying the palmaris longus and the ability of autografts to revascularize spontaneously, vascular anastomoses were not performed. Two autografts with and 2 autografts without neuroanastomosis were denervated 21 to 28 days before transplantation.

The autografts were studied 60 to 100 days following transplantation, except for one autograft without neuroanastomosis, which was studied after 183 days. Contractile properties were measured using a modified Fales muscle lever. ${ }^{21}$ Muscles were stimulated directly via stainless-steel needle electrodes inserted into the muscle belly near the proximal and distal tendons. Pulses were of $0.2-\mathrm{msec}$ duration. Muscle length and stimulator current were adjusted to give maximum isometric twitch tension. Maximum isometric tetanic tension was obtained by increasing the frequency of $0.2-\mathrm{msec}$ pulses delivered over a 200 -msec period. An index of fatigability was determined by stimulation with 0.2 -msec pulses at 70 pulses $/ \mathrm{sec}$ over a $200-\mathrm{msec}$ period, with one burst of impulses per second. The time in seconds required for the maximum isometric tetanic tension to decrease to $50 \%$ of the initial value was used as an index of fatigability. Responses were recorded on a storage oscilloscope and were then photographed. 'Time to peak twitch tension (TPT), one-half relaxation time $(1 / 2 \mathrm{RT})$, maximum isometric twitch tension $(\mathrm{Pt})$, and maximum isometric tetanic tension $(\mathbf{P o})$ were determined. The ratio of twitch to tetanic tension $(\mathrm{Pt} /$ Po) was calculated. Following measurement of contractile properties, autografts were isolated, removed, and weighed. Samples from the complete cross section of the belly of control and experimental muscles were placed in ice-cold physiologic saline solution for biochemical assays, or they were quick-frozen in isopentane cooled by dry icc for histochemical procedures. Results of histologic analysis of these atutografts are described elsewhere. ${ }^{15}$

For histochemical incubations, $14-\mu$ m-thick cross scctions were cut from the frozen blocks in a cryostat at $-20^{\circ} \mathrm{C}$. Sections of control and autografted muscles were incubated for succinic acid dehydrogenase (SDH), ${ }^{22}$ myofibrillar ATPase ${ }^{4}$ at pH 9.4, and capillary membrane ATPase ${ }^{16,13}$ activities. The capillary membrane ATPase was demonstrated in sections fixed in calcium formol and incubated at $\mathrm{pH} 9.4$ for $\mathrm{l} \mathrm{hr}$ in a myofibrillar ATPase medium to which $2.5 \mathrm{mM}$ parahydroxymercuribenzoate (PHMB) was added. This procedure completely inhibits myofibrillar ATPase and permits visualization of capillaries. The activity demonstrated is probably alkaline phosphatase.

Previous work has demonstrated that degenerative and regenerative events progress from the periphery toward the center of autografts, and that characteristics of regenerating muscle fibers in the periphery of autografts develop more quickly after transplantation than those of fibers toward the center of autografts. ${ }^{18}$ Therefore, at least six sample sites of each autograft cross section were selected from a peripheral zone representing approximately $30 \%$ of the total autograft cross section.

Methods for sampling, for classifying fibers, and for estimating capillarity have been previously described. ${ }^{16.18} \mathrm{~A}$ given fiber was categorized as fasttwitch or slow-twitch on the basis of myofibrillar ATPase activity, ${ }^{1,16-18}$ and as fatigue-resistant or fatigable on the basis of SDH activity. ${ }^{1,16}$ Fibers that were classified as fatigue-resistant had distinct SDH activity and subsarcolemmal aggregates of diformazan, especially near capillaries. Fibers that were classified as fatigable had very little SIH activity in subsarcolemmal regions. Both localization and intensity of activity were used in fiber classification. No studies have been done on the contractile properties of single motor units of autografts, making it difficult to verify classification of fibers as fasttwitch or slow-twitch. While we are aware of this limitation, we chose to classify fibers as fast-twitch or slow-twitch on the basis of myofibrillar ATPase activity for two reasons: to be consistent with previous work, and to quantify differentiation of myofibrillar ATPase in some regenerating muscle fibers. All slow-twitch (S) fibers are fatigue-resistant, but fast-twitch fibers can be classified as fast-twitch fatigue-resistant (FR) or fast-twitch fatigable (FF). Fiber area was determined by planimetry. Mean fiber area, percentage composition, fibers per square millimeter, capillaries per square millime- 
ter, and capillary-to-fiber ratio (capillaries per square millimeter divided by fibers per square millimeter) were calculated.

For biochemical studies, samples were blotted, weighed, and homogenized in 19 volumes of icecold $0.01 \mathrm{M}$ phosphate buffer $(\mathrm{pH} 7.4)$ using a Polytron homogenizer. Succinate oxidase activity of aliquots of the homogenate was determined by the method of Potter ${ }^{23}$ and expressed as $\mathrm{nl} \mathrm{O}_{2}$ used $\times$ mg homogenate protein ${ }^{-1} \times \min ^{-1}$. The protein content was determined by the method of Lowry et al. ${ }^{14}$

To find the number of sarcomeres per muscle fiber, entire muscles were removed, weighed, fixed to wooden splints, and immersed in isotonic saline containing $10 \%$ formalin. After several days, fixed muscles were immersed in $30 \%$ nitric acid for 2 to 3 days. ${ }^{28}$ This procedure dissolves connective tissue, exposing small bundles of muscle fibers. The nitric acid was then removed and replaced by $50 \%$ glycerol. The length of 20 muscle fibers was determined using a micrometer ocular under a dissecting microscope. After fiber-length measurement, the muscle fibers were wet mounted in $50 \%$ glycerol and were observed under oil immersion for measurement of sarcomere length. For each muscle, the length of a series of 10 sarcomeres was measured from each of 20 different fibers. The number of sarcomeres per muscle fiber was calculated as fiber length $\times$ sarcomere length ${ }^{-1}$. Insitu fiber length can be estimated by multiplying the number of sarcomeres per fiber by the desired in-situ sarcomere length.

Data from the muscles of each experimental group were pooled and statistical measures calculated. Differences between the means were tested by the t test, and significance was accepted at the $\mathrm{p}<0.05$ level. All statistical data are expressed as means \pm standard error of the mean.

\section{RESULtS}

Two autografts with anastomosis and two without anastomosis had been denervated three weeks before transplantation, but since these autografts were not significantly different from those that had not been predenervated, they were included for data analysis with the other autografts in their respective groups. Of eight palmaris longus muscles that had been transplanted orthotopically without neuroanastomosis, only three had regenerating muscle fibers at sacrifice. Data on fiber characteristics of autografts without anastomosis were obtained from these three autografts. The other five autografts without neuroanastomosis had no regenerating fi- bers and consisted of fibrous connective tissue. Of the autografts that had bcen transplanted without neuroanastomosis, none contracted when directly stimulated. The five autografts that formed fibrous slings from the origin to the insertion of the palmaris longus muscles provided support for the palmar fascia. In contrast, of the nine muscles that had been transplanted orthotopically with neuroanastomosis, all contained regenerating muscle fibers and all contracted when directly stimulated.

Figure 1 presents photomicrographs of a cross section of a monkey palmaris longus muscle that was transplanted orthotopically with neuroanastomosis. These photomicrographs demonstrate the peripheral-to-central gradient of muscle fiber maturity and the connective tissue core seen in orthotopic autografts. Early stages of regeneration are evidenced by the very small fibers toward the center of the cross section. At the periphery, larger muscle fibers are present; except for the central nuclei, these fibers have a histochemical appearance similar to that of control muscle fibers.

Initially, all regenerating fibers have the histochemical characteristics of FF fibers, but $S$ and FR fibers differentiate at a later time. ${ }^{16}$ Autografts with neuroanastomosis showed differentiation of muscle fibers into the three fiber types normally seen in limb skeletal muscles (table 1). The predominance of fatigable fibers in the autografts relative to control muscles is consistent with the measured succinate oxidase values: $43 \pm 12$ and $38 \pm$ $19 \mathrm{nl} \mathrm{O}_{2} \times \mathrm{mg}_{\text {protein }}{ }^{-1} \times \min ^{-1}$, respectively, for autografts with or without neuroanastomosis, as

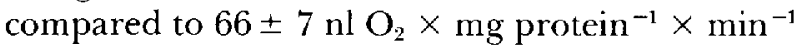
for control palmaris longus muscles. Thus, at sacrifice, the autografts had only $65 \%$ of the oxidative capacity of the controls. The percentage composition of skeletal muscle fibers in autografts, both with and without neuroanastomosis, is significantly different from that of control muscles; but the composition of autografts with neuroanastomosis did not differ significantly from that of the three autografts without neuroanastomosis in which muscle fibers had regenerated.

Mean fiber areas are smaller in these autografts than in control muscles, especially FF fibers (table 2). No significant difference in mean fiber area was observed between muscles transplanted with and those transplanted without neuroanastomosis. The frequency distribution of fiber area for autografts with neuroanastomosis (fig. 2) shows a unimodal distribution, with a peak at $600 \mu \mathrm{m}^{2}$ at 65 and 83 days after transplantation. At 100 days, although many $600 \mu \mathrm{m}^{2}$ fibers arc still evident, the height 


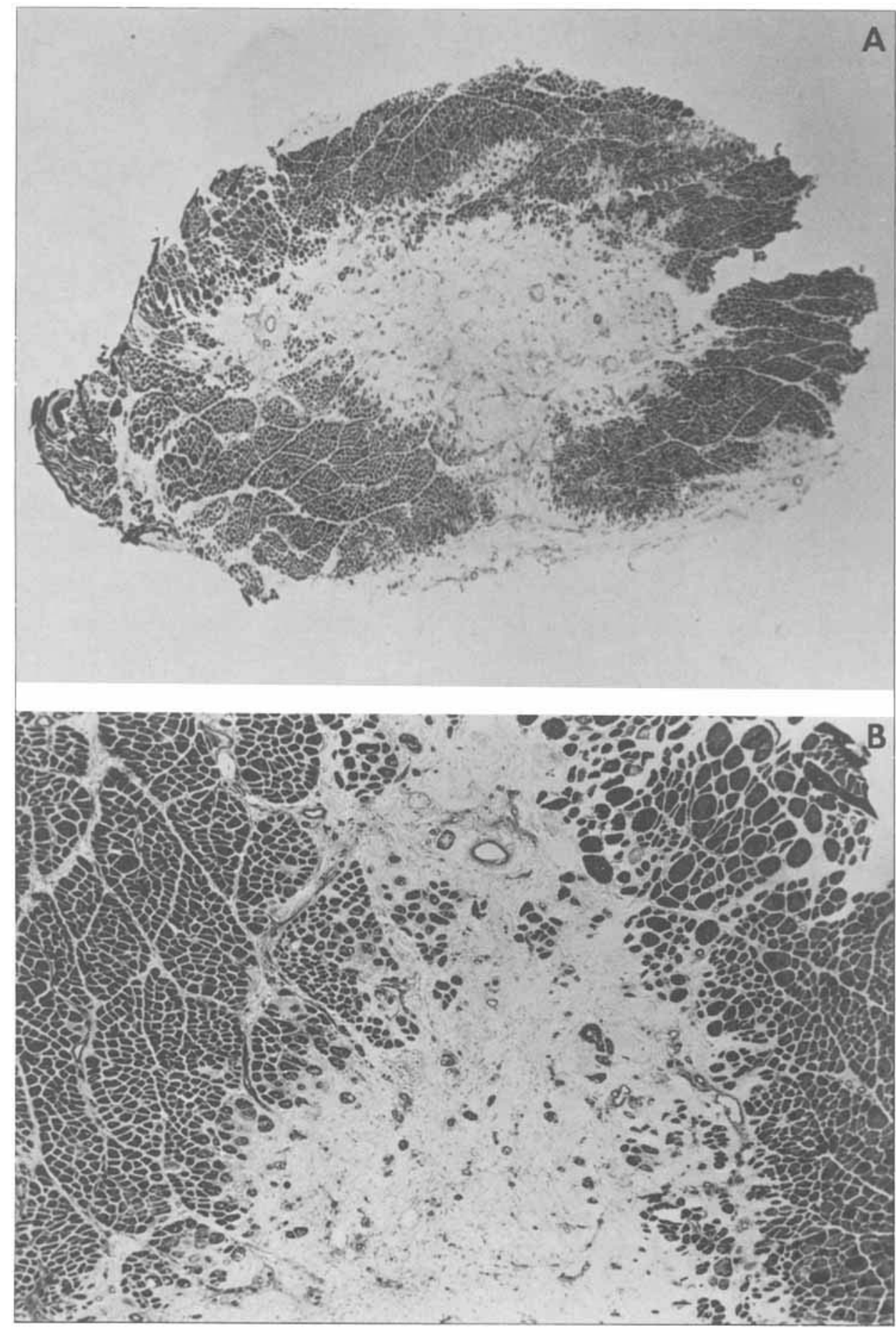

Figure 1. Cross section of a palmaris longus muscle 83 days after orthotopic transplantation with neuroanastomosis. Incubated for demonstration of myofibrillar ATPase.

(A) Magnification $\times 14$

(B) Magnification $\times 56$. of the $600 \mu \mathrm{m}^{2}$ peak is diminished relative to the 83-day autograft, and the distribution includes fibers with cross-sectional areas of up to $3,000 \mu \mathrm{m}^{2}$.

Autografts begin to revascularize spontaneously shortly after transplantation; but 60 to 100 days after transplantation, they still have fewer capillaries adjacent to individual muscle fibers (table 3 ), fewer capillaries per square millimeter, and a lower capillary-to-muscle-fiber ratio (table 4) than control muscles. With respect to these variables, no significant difference was found between autografts with neuroanastomosis and autografts with- 


\begin{tabular}{|c|c|c|c|}
\hline Muscles & $S$ & $\mathrm{FR}$ & FF \\
\hline Controls $(N=11)$ & $14.58 \pm 2.13$ & $35.28 \pm 5.26$ & $49.95 \pm 5.08$ \\
\hline $\begin{array}{l}\text { Orthotopic transplants without } \\
\text { neuroanastomosis }(N=3)^{\circ}\end{array}$ & $0.97 \pm 0.97^{c}$ & $3.40 \pm 1.14^{\circ}$ & $95.63 \pm 0.32^{c}$ \\
\hline $\begin{array}{l}\text { Orthotopic transplants with neuroanastomosis } \\
(N=9)\end{array}$ & $1.48 \pm 1.24^{c}$ & $7.21 \pm 4.97^{c}$ & $91.31 \pm 6.18^{c}$ \\
\hline
\end{tabular}

\begin{tabular}{lccc}
\multicolumn{4}{c}{ Table 2. Mean muscle fiber areas $\left(\mu \mathrm{m}^{2}\right)$ of control and transplanted palmaris longus muscles. } \\
\hline Muscles & $\mathrm{S}$ & $\mathrm{FR}$ & $\mathrm{FF}$ \\
\hline Control $(\mathrm{N}=11)$ & $2,874 \pm 341$ & $3,758 \pm 458$ & $4,548 \pm 478$ \\
Orthotopic transplants without & $2,228 \pm 470$ & $2,317 \pm 617$ & $1,474 \pm 746^{\mathrm{b}}$ \\
$\quad$ neuroanastomosis $(\mathrm{N}=3)$ & & & \\
Orthotopic transplants with neuroanastomosis & $1,374 \pm 430^{\mathrm{b}}$ & $1,749 \pm 499^{\mathrm{b}}$ & $1,095 \pm 156^{\mathrm{b}}$ \\
$(\mathrm{N}=9)$ & & & \\
\hline
\end{tabular}

aalues given as mean \pm SEM.

'Significantly different from control values $(p<0.05)$

out neuroanastomosis in which muscle fibers had regenerated.

Monkey palmaris longus autografts with neuroanastomosis could produce contraction and develop tension when stimulated directly. Autografts without neuroanastomosis did not contract even when stimulated with a voltage well in excess of that required for maximum stimulation of control muscles or autografts with neuroanastomosis. Representative records of tension development during isometric twitch and tetanic contractions are shown in figure 3. Table 5 delineates the mean contractile properties of 9 autografts with neuroanastomosis and 15 control palmaris longus muscles. During isometric twitches, both tension development and relaxation were slower for autogralts than for control muscles. For autografts, time to peak tension was $126 \%$, and one-half relaxation time $173 \%$, of control values. The control and autografted muscles were not freed up as much as is usually done for in-situ preparation; consequently, time to peak tension may be slightly slower than is usually reported. However, the data are comparable. Compared to control values, active tension developed by autografts was only $6 \%$ for isometric twitches and $5 \%$ for isometric tetanus, but the ratio of twitch to tetanic peak tension was not significantly different for autografts than for controls. Auto- grafted muscles weighed $1.47 \pm 0.36 \mathrm{~g}$, compared to $2.55 \pm 0.29 \mathrm{~g}$ for control muscles. However, up to $80 \%$ of the cross-sectional area of autografts was noncontractile connective tissue in the central core. Additionally, there was considerable infiltration of connective tissue between fascicles of regenerating muscle fibers, and many of the regenerating fibers were very small and immature. We therefore estimate that the contractile mass of autografts averages less than $10 \%$ of that of control muscles. Thus, it appears that the tension development normalized for the total fiber cross-sectional area of autografted muscles may approach control values.

Autografted muscles shortened only $36 \%$ as much as control muscles. Part of this difference is due to the shorter fibers of autografts in relation to those of control muscles. Our estimate of 4,580 \pm 50 sarcomeres per autografted muscle fiber is $53 \%$ of the control muscle value of $8,680 \pm 470$ sarcomeres per fiber.

\section{DISCUSSION}

A number of investigators ${ }^{6,9,10,26,27}$ have indicated that denervation of the donor muscle two to four weeks before transplantation is a prerequisite for successful transplantation. It was the interpretation of these investigators that prior denervation caused a reduction of the metabolic requirements 


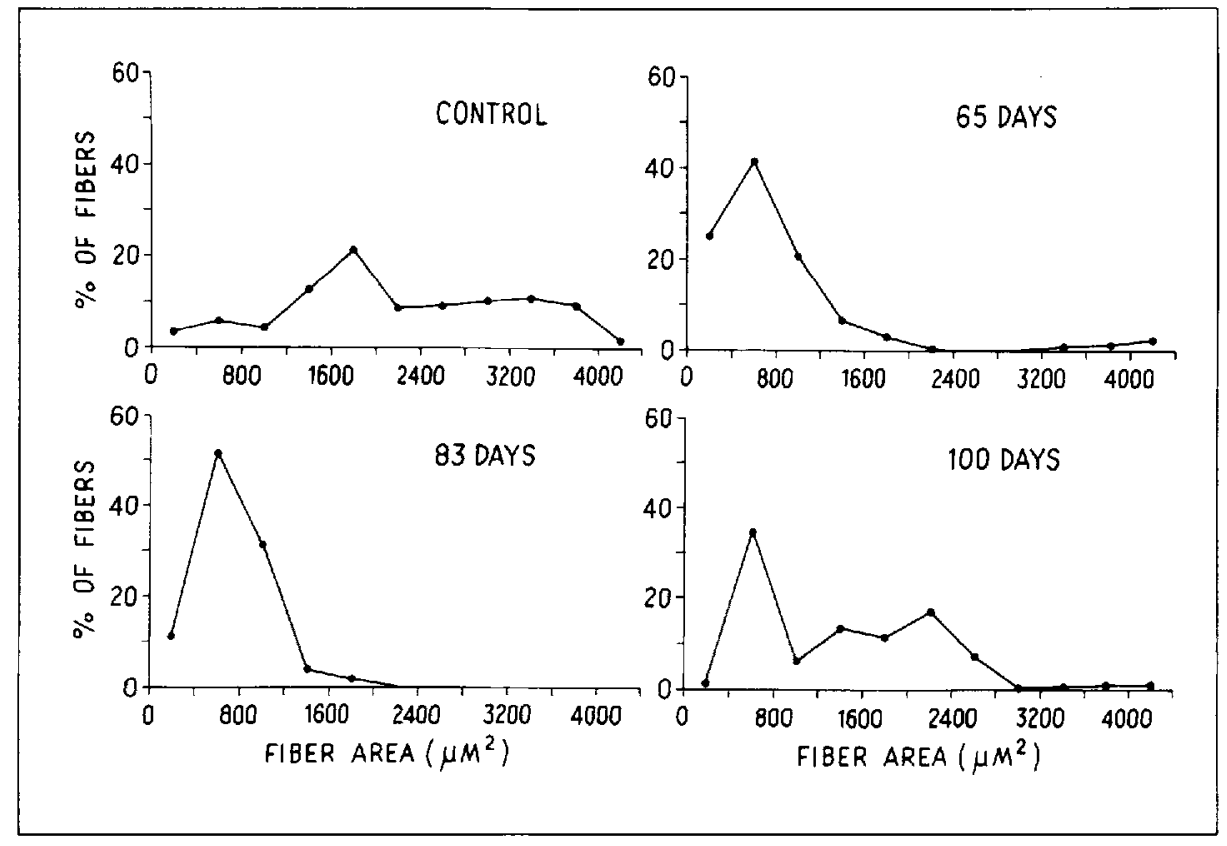

Figure 2. Frequency distributions of fiber areas for control muscles and for selected autografted muscles with neuroanastomosis 65,83 , and 100 days after transplantation.

of the tissue, which enabled the autograft to survive the ischemia that resulted from complete isolation of the muscle. ${ }^{24}$ However, successful transplantation without prior denervation has been observed for muscles of rats ${ }^{2,29}$ and cats, $, 51,20$ and is now described for palmaris longus muscles of monkeys.
Of major importance in the study of autografted muscles is the question whether muscle fibers in autografts are surviving original muscle fibers or regenerating fibers. Resolution of this question requires analysis of a series of autografts during the first weeks following transplantation. We have shown previously that very few original

Table 3. Number of capillaries adjacent to muscle tibers of control and transplanted palmaris longus muscles. ${ }^{a}$

\begin{tabular}{llll}
\hline Muscles & $S$ & FR & FF \\
\hline Controls $(N=11)$ & $4.34 \pm 0.30$ & $4.54 \pm 0.33$ & $4.15 \pm 0.27$ \\
Orthotopic transplants without & $2.40 \pm 0.00^{\circ}$ & $1.94 \pm 0.53^{\mathrm{b}}$ & $1.21 \pm 0.57^{\mathrm{b}}$ \\
$\quad$ neuroanastomosis $(\mathrm{N}=3)$ & & & \\
Orthotopic transplants with neuroanastomosis & $0.96 \pm 0.04^{\mathrm{b}}$ & $2.00 \pm 0.61^{\mathrm{b}}$ & $1.01 \pm 0.20^{\mathrm{b}}$ \\
$(\mathrm{N}=9)$ & & & \\
\hline
\end{tabular}

avalues given as mean + SEM.

- Significantly different from control values $(p<0.05)$.

\begin{tabular}{lcc} 
Table 4. Capillary density and capilary-io-muscle-fiber ratio for control and transplanted muscles. & \\
\hline Muscles & $\begin{array}{c}\text { Capillaries/ } \\
\mathrm{mm}^{2}\end{array}$ & $\begin{array}{r}\text { Capillary-to- } \\
\text { muscle-fiber ratio }\end{array}$ \\
\hline Controls $(\mathrm{N}=11)$ & $480 \pm 60$ & $1.80 \pm 0.16$ \\
Orthotopic transplants without & $199 \pm 6^{\mathrm{b}}$ & $0.58 \pm 0.22^{\mathrm{b}}$ \\
$\quad$ anastomosis $(\mathrm{N}=3$ ) & $258 \pm 62^{\mathrm{b}}$ & $0.43 \pm 0.10^{\mathrm{b}}$ \\
Orthotopic transplants with neuroanastomosis & & \\
$(\mathrm{N}=9)$ &
\end{tabular}

avalues given as mean $\pm S E M$.

"Significantly different from control values $(p<0.05)$. 
Figure 3. Isometric twitch and tetanus recordings from a control and an autografted muscle.

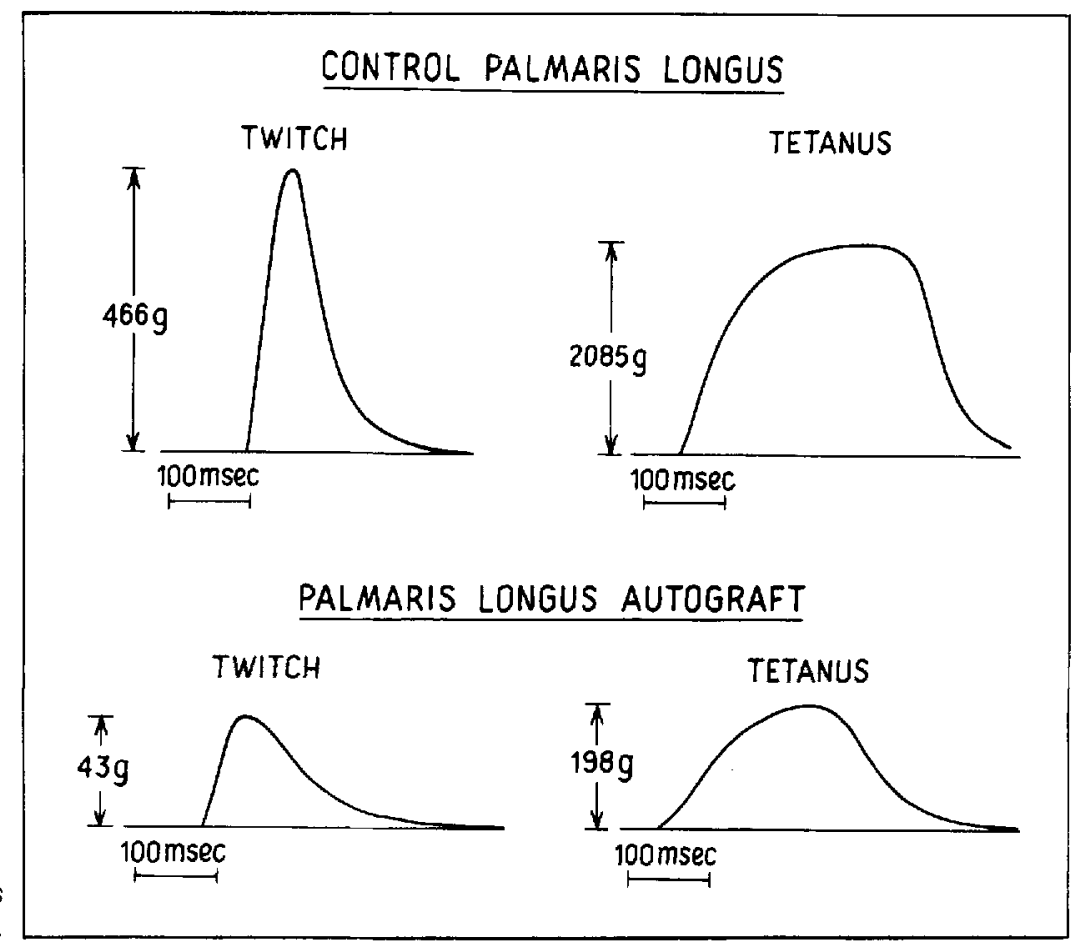

muscle fibers survive the ischemia following transplantation of extensor digitorum longus muscles of cats. ${ }^{18.20}$ While we have not done definitive analysis for surviving fibers in autografts of palmaris longus muscles of monkeys, we presume, on the basis of the data from cats, that an insignificant number of original muscle fibers survive, and that essentially all fibers observed are regenerating.

Successful innervation of regenerating muscle fibers has been observed directly in autografts by demonstration of motor endplates. ${ }^{7.11}$ Innervation of muscle fibers in autografts may occur from adjacent nerve trunks or from branching of nerves to adjacent muscles. In the absence of innervation, neither developing ${ }^{24}$ nor regenerating ${ }^{3,19}$ muscle fibers differentiate. Failure of reinnervation is manifested in autografts by failure of regenerating muscle fibers to differentiate into the three types of muscle fibers normally seen in skeletal muscle, and also by failure of muscle fibers to hypertrophy. We interpret differentiation of regenerating muscle fibers into different fiber types as evidence of reinnervation of autografts.

Transplantation without neuroanastomosis results in good restoration of structure and function with regeneration of muscle fibers in the extensor digitorum longus muscle of cats; $;^{5,18,20}$ but in the palmaris longus muscle of monkeys, we have found that transplantation without neuroanastomosis often results in a fibrous sling with no contractile activity. The recipient sites for transplantation are quite different. In the cat hindlimb, the profundus nerve lies immediately beneath the extensor digitorum longus muscle. When it is cut, it retracts to the space beneath the muscle belly. In contrast, the motor nerve to the palmaris longus muscle in monkeys enters the muscle perpendicular to its belly; and, when it is cut, it retracts between the flexor digitorum profundus and flexor carpi radialis muscles. Thus, a direct source for neurotization is further removed from autografts of monkey palmaris longus autografts muscles than from autografts of cat extensor digitorum longus muscles. We conclude that spontaneous innervation of monkey palmaris longus autografts is possible but does not readily occur. The failure of substantial regeneration of muscle fibers in five of the eight palmaris longus muscles transplanted without neuroanastomosis in monkeys is consistent with this conclusion.

In contrast to palmaris longus muscles transplanted without neuroanastomosis, palmaris longus muscles transplanted with motor nerve repair regenerate and have characteristics similar to transplanted extensor digitorum longus muscles of cats. ${ }^{5,18,20}$ Sixty to 100 days after transplantation, palmaris longus muscles transplanted with neuroanastomosis have a central core of connective tis- 
sue, bundles of small regenerating muscle fibers with central nuclei, and a peripheral-to-central gradient of muscle fiber maturity. These observations suggest that the peripheral-to-central progression of degenerative and regenerative events previously observed in transplanted cat extensor digitorum longus muscles $s^{5,18,20}$ occur in the transplanted monkey palmaris longus muscles.

The predominance of $\mathrm{FF}$ fibers in the palmaris longus autografts in monkeys is consistent with results from the extensor digitorum longus autografts in cats 60 to 100 days after transplantation. ${ }^{18}$ All regenerating fibers begin with an FF enzymatic profile, further differentiating only after the establishment of functional innervation. Nerve fibers from the anastomosed motor nerve penetrate the autografts and were observed near regenerating muscle fibers. ${ }^{15}$ The histochemical data show differentiation of myofibrillar ATPase and development of oxidative capacity by regenerating fibers. In a given muscle, changes in succinate oxidase or succinic acid dehydrogenase activity are indicators of alteration in the frequency of recruitment of its skeletal muscle fibers. ${ }^{17}$ Spontaneous EMG activity has been observed in heterotopically transplanted monkey palmaris longus muscles at comparable times after transplantation (G. C. Gorniak, personal communication). All of these observations indicate that autografts are recruited. However, the succinate oxidase activity of homogenates, the SDH activity of fibers, the proportion of slow-twitch fibers, and the capillarity of the palmaris longus autografts are all less than what has been observed in control palmaris longus muscles. We conclude that the frequency of usage of the autografts by the animal does not recover to control levels dur-

Table 5. Contractile properties of control muscles and autografted muscles with neuroanastomosis. ${ }^{2}$

\begin{tabular}{lcc}
\hline Properties $^{b}$ & $\begin{array}{c}\text { Control muscles } \\
(\mathrm{N}=15)\end{array}$ & $\begin{array}{c}\text { Autografts } \\
(\mathrm{N}=9)\end{array}$ \\
\hline TPT (msec) & $41.77 \pm 1.90$ & $52.69 \pm 4.37^{\mathrm{c}}$ \\
$1 / 2 \mathrm{RT}(\mathrm{msec})$ & $32.56 \pm 3.30$ & $56.19 \pm 4.46^{\mathrm{c}}$ \\
Pt $(\mathrm{g})$ & $475 \pm 43$ & $26 \pm 6^{\mathrm{c}}$ \\
Po $(\mathrm{g})$ & $2.152 \pm 237$ & $106 \pm 23^{\mathrm{c}}$ \\
Pt/Po & $0.23 \pm 0.02$ & $0.26 \pm 0.04$ \\
Shortening $(\mathrm{mm})$ & $11.44 \pm 1.07$ & $4.08 \pm 0.54^{c}$ \\
$\%$ shortening & $49 \pm 5$ & $33 \pm 17$ \\
\hline
\end{tabular}

avalues given as mean \pm SEM.

'TPT $=$ time to peak twitch tension; $R T=$ relaxation time

$\mathrm{Pt}=$ maximum isometric twitch tension; $\mathrm{PO}=$ maximum isometric tetanic tension.

cSignificantly different from controt values $(p<0.05$ ). ing the first 100 days after transplantation.

The mean fiber area of the autografted muscles is less than that of control palmaris longus muscles. Sixty days after transplantation, autografts contain predominantly small fibers in the early stages of regeneration. In older autografts (100 days after transplantation), there are fewer small fibers and increasing proportions of fibers with cross-sectional areas similar to control palmaris longus muscle fibers. Thus, there is evidence of growth of individual fibers 60-100 days after transplantation. These results are similar to autografts of extensor digitorum longus muscles of cats during this time interval, ${ }^{18}$ Based on data from our autografts in cats, we would expect fibers in autografts of monkey palmaris longus muscles to reach or exceed control muscle fiber area if they were left to mature longer. The fibers of autografts of extensor digitorum longus muscles of cats require 170 days to hypertrophy to greater than control mean fiber area. ${ }^{18}$

Autografts with neuroanastomosis have contractile properties indicating recovery toward control function. However, neither the development of isometric tension nor the rate of relaxation is as rapid for autografted as for control muscles. The longer time to peak tension is not consistent with the greater proportion of fast-twitch fibers of autografted muscles. Whether this slower development of tension is an intrinsic characteristic of the contractile machinery of regenerating muscle fibers, whether it results from mechanical interference by the excess connective tissue, or whether it perhaps represents some other metabolic or physiologic deficiency is not clear. Similarly, we have no data to elucidate the basis for the slower relaxation of autografts. These observations are, however, similar to those made previously on autografts of cat extensor digitorum longus muscles. ${ }^{5}$

Small muscle fibers and a central core of noncontractile connective tissue result in low development of tension by the autografts, but tension normalized for total fiber cross-sectional area appears normal. A large noncontractile central core, but near-normal tension per fiber cross section, is consistent with our previous experience with autografts of cat extensor digitorum longus muscles 60 to 100 days after transplantation. ${ }^{5}$ Both absolute and relative shortening are reduced for autografts in comparison to control muscles. This reduced shortening seems to be partially attributable to the shorter muscle fibers in autografts than in control muscles. 
Successful regeneration of monkey palmaris longus muscles occurs when the muscles are orthotopically transplanted without prior denervation. While regenerating muscle fibers are observed in a few autografts without neuroanastomosis, the success rate for regeneration is significantly improved by repair of the motor nerve at the time of transplantation. Neuroanastomosis can significantly benefit skeletal muscle transplantation by providing a readily accessible source of innervation in the recipient site of an autografted muscle.

\section{REFEAENCES}

1. Burke RE, I evine DN, Zajac FE III, Tsairis P, Engel WK: Mammalian motor units: physiological-histochemical correlates in three types in cat gastrocnemius. Science 174:709712,1971

2. Carlson BM, Gutmann E: Regeneration in free grafts of normal and denervated muscles in the rat: morphology and histochemistry. Anat Rec 183:47-62, 1975 .

3. Carlson BM. Gutmann E: Contractile and histochemical properties of sliced muscle grafts regencrating in normal and denervated rat limbs. Exp Neurol 50:319-329, 1976.

4. Chayen J, Bitensky L, Butcher RG: Practical Biochemistry. New York, John Wiley \& Sons, 1973, pp. 122-124.

5. Faulkner JA, Maxwell LC, Mufti SA, Carlson BM: Skeletal muscle fiber regeneration following heterotopic autotransplantation in cats. Life Sci 19:289-296, 1976.

6. Freilinger G, Holle H, Mamoli B: Free muscle transplants for anal sphincter reconstruction in pigs. Chir Plastica 2:133$141,1974$.

7. Gruber H, Freilinger G, Holle J, Mayr R: Motor endplates in autologous muscle transplants. Experientia 20:1191-1192, 1974.

8. Harii $\mathrm{K}$, Ohmori $\mathrm{K}$, Torii $\mathrm{S}$ : Free gracilis muscle transplantation, with microneurovascular anastomoses for the treatment of facial paralysis. Plast Reconstr Surg 57:133143,1976 .

9. Hakelius I.: Transplantation of free autogenous muscle in the treatment of facial paralysis. Scand J Plast Reconstr Surg 8:220-230, 1974.

10. Hakelius L: Free autogenous muscle transplantation in two cases of total anal incontinence. Acta Chir Scand 141:69-75, 1974.

11. Hakelius L, Nystrom B: Histochemical studies of endplate formation in free autologous muscle transplants in cats. Scand J Plas Reconstr Surg 9:9-14, 1975.

12. Hakelius L, Nystrom B, Stålberg E: Histochemical and neurophysiological studies of autotransplanted cat muscle Scand J Plast Reconstr Surg 48:11-27, 1971.

13. Kubo T, Ikuta $Y$, Tsuge K: Free muscle transplantation in dogs by microneurovascular anastomoses. Plast Reconstr Surg 57:495-501, 1976

14. Lowry OH, Rosebrough NJ, Faro AL, Crandall RJ: Protein measurement with the Folin phenol reagent. $J$ Biol Chem 193:265-275, 1951.

15. Markley JM Jr, Faulkner JA, Carlson BM: Regeneration following transplantation in monkeys. Plast Reconstr Surg
$62: 415-422,1978$

16. Maxwell LC, Barclay JK, Mohrman DE, Faulkner JA: Physiological characteristics of skeletal muscles of dogs and cats. Am J Physiol 233:C14-C18, 19 ל7.

17. Maxwell LC, Faulkner IA, Lieberman DA: Histochemical manifestations of age and endurance training. $A m J$ Physiol $224: 356-361,1973$

18. Maxwell LC, Faulkner JA, Mufti SA, Turowski AM: The frec autografting of entire limb muscles in the cat: histochemistry and biochemistry. $J$ Appl Physiol 44:431-437, 1978 .

19. Mong FSF: Histological and histochemical studies on the nervous influence on minced muscle regeneration of triceps surae of the rat. J Morphol 151:451-462, 1977.

20. Mufti SA, Carlson B M, Maxwell LC, Faulkner JA: The free autografting of entire limb muscles in the cat: morphology. Anat Rec 188:417-429, 1977.

21. Murphy RA, Beardsley AC: Mechanical properties of the cat soleus muscle in situ. Am J Physial 227:1008-1013, 1974.

22. Nachlas J, Tsou MK, deSouza E, Cheng C, Seligman AM Cytochemical demonstration of succinic dehydrogenase by the use of a new p-nitrophenyl substituted ditetrazole. $I$ Historhem Cytochem 12:740-743, 1964.

23. Potter VR: The homogenate technique. In Umbreit WW, Burris RH, Stauffer JF (Editors): Manometric Techniques. Minneapolis, Burgess Publishing, 1964, pp 159-176.

24. Shafiq SA, Asiedre SA, Milhorat AT: Effect of neonatal ncurectomy on differentiation of fiber types in rat skeletal muscle. Exp Neurol 35:529-540, 1972.

25. Schenck RR: Rectus femoris muscle and composite skin transplantation by microneurovascular anastomoses for avulsion of forearm muscles: a case report. J Hand Surg $3: 60-69,1978$.

26. Studitsky AN, Bosova NN: Development of atrophic muscular tissue in conditions of transplantation in place of mechanically damaged muscles. Arkh Anat Gistol Embriol 369:18-32, 1960 .

27. Thompson N: Autogenous free grafts of skeletal muscle Plast Reconstr Surg 48:11-27, 1971

28. Williams PE, Gold spink G: Longitudinal growth of striated muscle fibers. J Cell Sci 9:751-767, 1971 .

29. Zhenevskaya RP, Rumyantseva Proshlyakova EV: Regenerative processes in a transplant of untreated muscle of young rats. Zhur Obsch Biol 26:569-576, 1965. 